Int. J. Speleol. 4 (1972), pp. 67-75.

\title{
La Migration des Trichoptères cavernicoles. Mise en évidence par piégeage lumineux**
}

\author{
YVETTE BOUVET *
}

Les grottes renferment une faune très intéressante dont la zonation dépend de l'appartenance plus ou moins grande des groupes considérés au milieu cavernicole.

La faune pariétale des entrées de grottes se comporte à cet égard de manière très caractéristique, et l'on observe toute une série d'Arthropodes trogloxènes ou troglophiles qui séjournent saisonnièrement dans la zone de transition entre le milieu souterrain profond et le domaine épigé (Jeannel, 1943; Motas et Col., 1967).

Les Trichoptères cavernicoles appartiennent à cette association caractéristique de l'entrée des cavités souterraines et leur cycle biologique se trouve marqué par le séjour estival des imagos sous terre.

La motivation de ce séjour souterrain n'est pas très bien définie et l'on sait simplement que les femelles de Trichoptères subissent une diapause ovarienne au cours de la phase cavernicole de leur vie imaginale (Bouvet, 1971). On a d'ailleurs longtemps admis que les Trichoptères périssaient sous terre, ce milieu fermé fonctionnant comme un piège à leur égard. Il faut cependant admettre qu'aucune preuve expérimentale n'étayait cette hypothèse.

Une méthode de piégeage lumineux vient donc d'être utilisée pour tenter de résoudre ce problème de la sortie des Trichoptères cavernicoles des grottes à la fin de l'été, sortie qui correspondrait alors à un envol vers les lieux de pontes épigés.

Deux conditions se révèlent nécessaires pour démontrer la sortie des Trichoptères adultes vers l'extérieur des grottes:

- marquer les individus sous terre

- capturer ces mêmes individus à l'extérieur.

La marque, apposée sur chaque insecte capturé à la main, se compose d'une légère tache de vernis à ongles coloré disposée à l'avant de l'aile antérieure droite. Une telle technique avait déjà été employée pour l'étude des Trichoptères cavernicoles par Bitsch et Frochot (1962), Bournaud et Gautheron-Duranthon (1969). Le vernis s'applique facilement au pinceau; il suffit de ne pas coller les ailes et de ne pas trop les charger pour qu'aucune gène ne se manifeste chez les Trichoptères qui se déplacent alors normalement. La méthode conduit parfois à une sous-estimation dans l'expression du pourcentage de recapture, certains insectes pouvant perdre leur marque.

Les Trichoptères se capturent facilement la nuit à l'aide d'un piège lumineux. Le piège utilisé lors de cette expérience (Fig. 1), construit suivant les plans de Fontaine (1971), fonctionne sur une batterie d'accumulateurs de 12 volts. Le tube fluores-

* Laboratoire de Biologie souterraine. Université Claude-Bernard, 16 Quai Claude Bernard, Lyon $7^{\circ}$.

** Collaboration technique: Roger LAURENT 


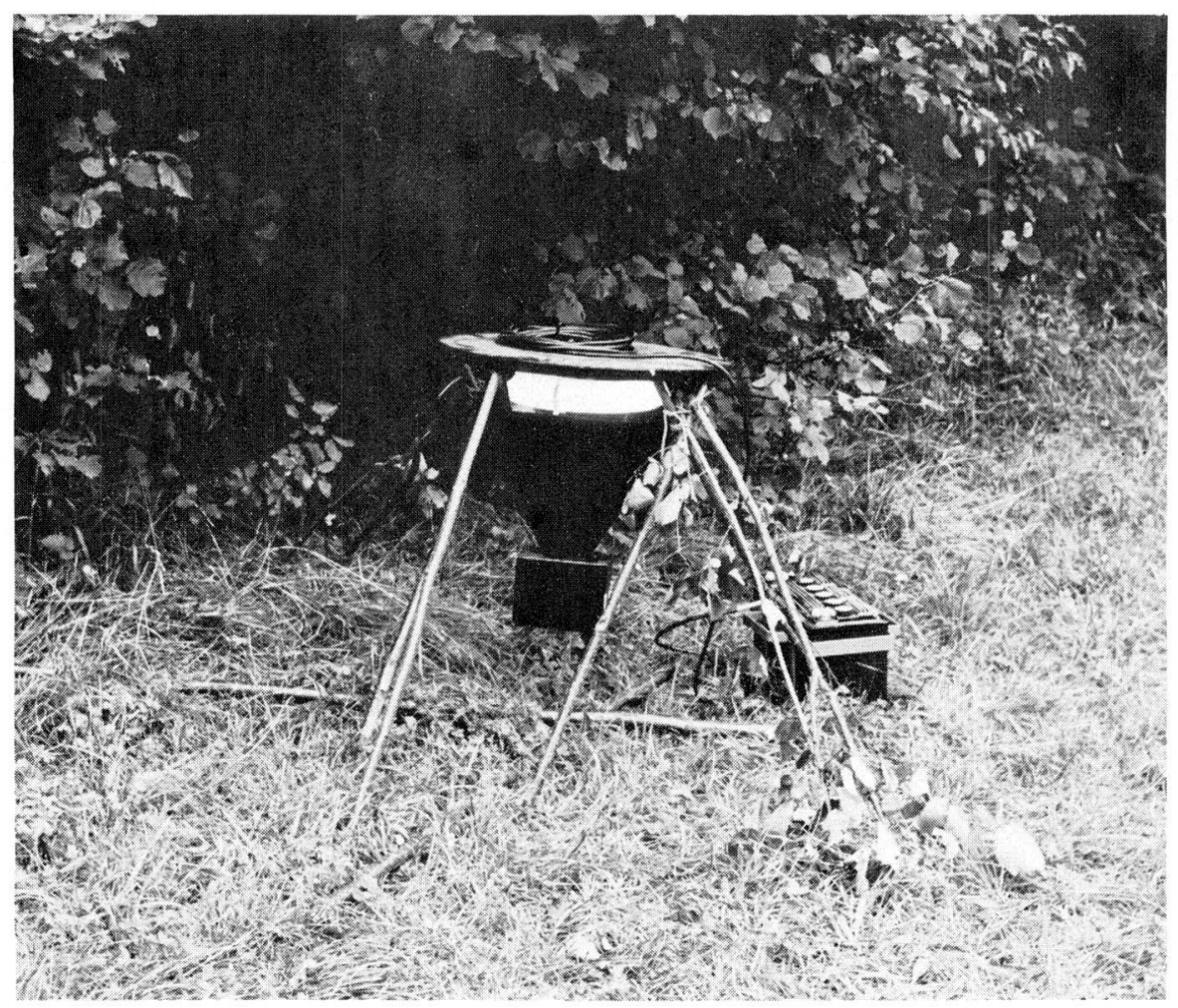

Fig. 1. Piège lumineux et système d'alimentation électrique.

cent en anneau, d'une puissance de 32 watts, émet une lumière dont la longueur d'ondes est comprise entre 3.000 et 6.500 angströms (Fig. 2).

La réussite de l'expérience dépend du complexe topographique choisi; il doit en effet comprendre:

- une grotte abritant une population de Trichoptères suffisamment abondante;

- un ruisseau épigé où l'on retrouve les larves des espèces cavernicoles de la grotte;

Ces deux biotopes, situés à faible distance l'un de l'autre, permettent d'éviter une trop grande "dilution" des individus marqués par les Trichoptères issus d'autres biotopes. Le lieu choisi se situe à Petit-Corent, commune de Simandre-sur-Suran (Ain), dans la grotte de la source de la Dhuys, pointée sur la carte I.G.N. au 20.000ème Nantua no. I; X: 839,10 Y: 141,09 Z: 483 m.

Cette grotte se compose de deux petites salles qui peuvent être noyées lors des crues de la circulation d'eau souterraine. On y rencontre une abondante population de Trichoptères (300 à 500 individus pendant l'été). La température est de $9,5^{\circ} \mathrm{C}$ 


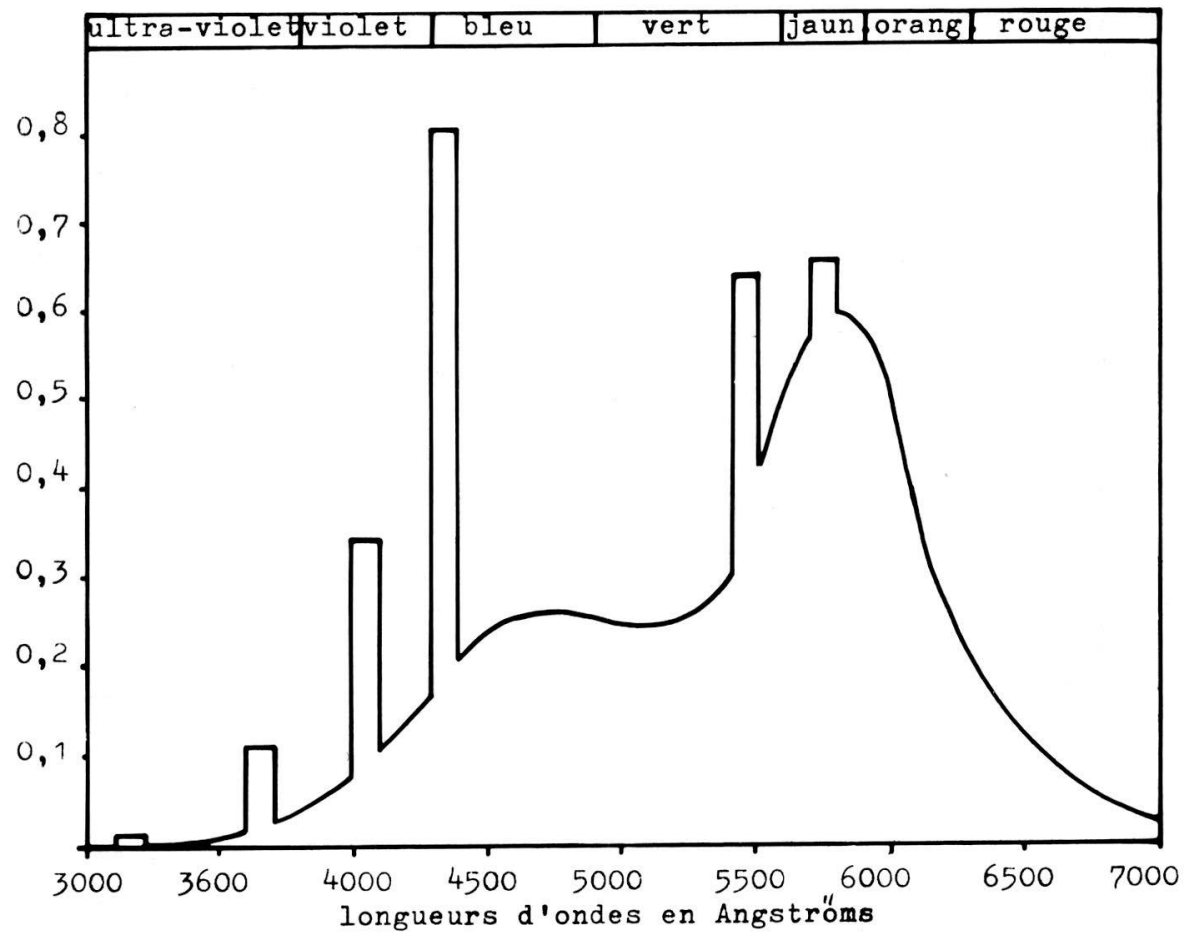

Fig. 2. Spectre d'émission de la lampe équipant le piège lumineux.

$\pm 0,5^{\circ} \mathrm{C}$ en septembre, dans un milieu très humide (100\% d'humidité). Un ruisseau temporaire en sort, dans lequel on retrouve, dès le mois d'octobre, des larves de Trichoptères des mêmes espèces que celles de la grotte.

Le ler septembre 1970, 230 Trichoptères sont marqués de la manière indiquée précédemment, à l'intérieur de la grotte. Ils se tiennent dans une zone à obscurité complète. Les individus marqués se répartissent de la manière suivante:

Stenophylax permistus: $570^{\circ}+152 q$

Stenophylax mitis: $\quad 90^{\circ}+9 q$

Micropterna nycterobia: $30^{\circ}$

Le piège lumineux, installé à dix mètres de l'entrée de la grotte, se trouve masqué de cette entrée par de gros blocs de pierre formant ressaut. Cette disposition évite la pénétration dans la grotte d'un rayon de lumière émis par le piège qui alors aurait attiré les Trichoptères vers l'extérieur. Cette précaution prise, les individus marqués capturés dans le piège lumineux sortent bien activement de la grotte.

Le piège fonctionne du 2 septembre au 2 octobre 1970, avec quatre nuits 


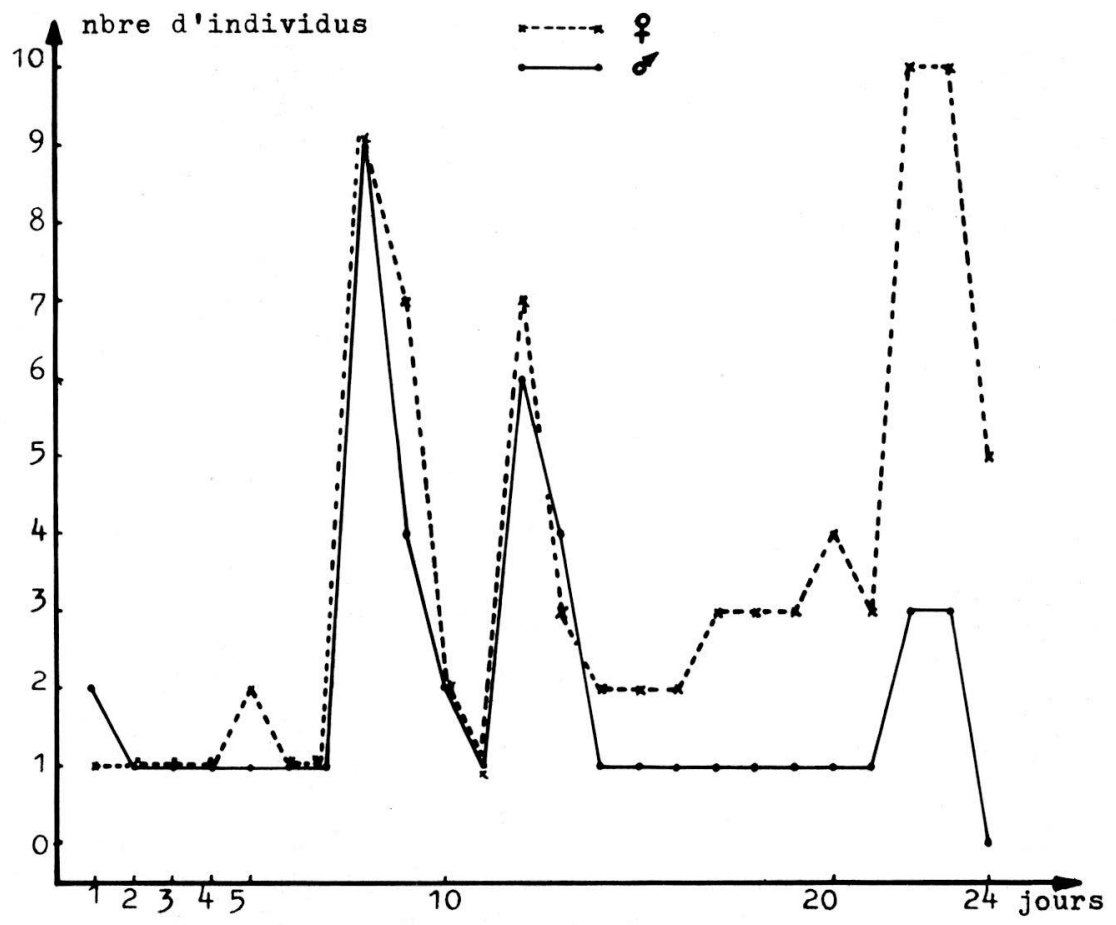

Fig. 3. Nombre d'individus capturés par sexe. En ordonnée, l'unité utilisée correspond à un groupe de 5 individus.

d'interruption du 25 au 29 septembre (fig. 5). Au cours de cette période, dix individus marqués sont récoltés dans le piège; ce faible nombre de recaptures s'explique de diverses manières. D'une part, les Trichoptères peuvent soit perdre leur marque, soit se faire dévorer par les prédateurs habituels avant leur sortie de la grotte; d'autre part, ils s'échappent parfois de l'entonnoir du piège avant d'avoir atteint le liquide fixateur contenu dans le collecteur; ils peuvent enfin se disperser vers le ruisseau sans se diriger sur le piège.

Tableau des recaptures de Trichoptères

\begin{tabular}{|c|c|c|c|c|c|c|}
\hline espèces & $4 / 5-9$ & $7 / 8-9$ & $11 / 12-9$ & $12 / 13-9$ & $15 / 16-9$ & 18/19-9: 1971 \\
\hline Stenophylax permistus & $1 \delta^{*}$ & $2 q$ & $1 \delta+1 q$ & $2 \uparrow$ & & 10 \\
\hline Stenophylax mitis & & & 19 & & 19 & \\
\hline
\end{tabular}


Symboles utilisés: $M=$ mouillé, $H=$ humide, $S=\sec , V=$ vent,$\quad v=$ brise,$W=$ vent nul, $\otimes=$ pluie, $\zeta_{1}=$ orage, $==$ brume, $\equiv=$ brouillard.

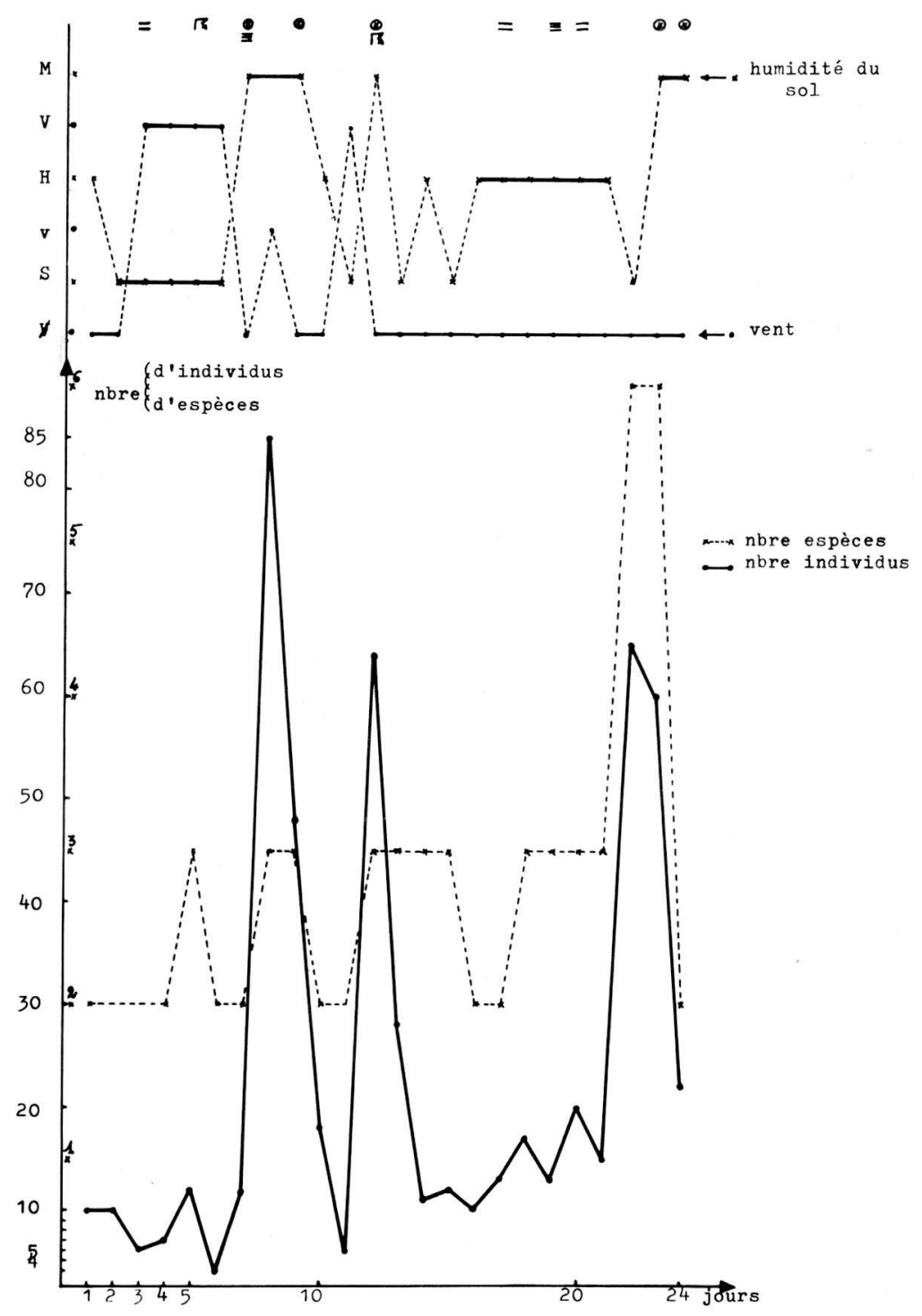

Fig. 4. Influence des facteurs climatiques sur le nombre de Trichoptères capturés, en fonction du temps. 


\begin{tabular}{|c|c|c|c|c|c|c|c|c|c|c|c|c|}
\hline $\mathrm{N}^{\circ}$ piège & 1 & 2 & 3 & 4 & 5 & 6 & 7 & 8 & 9 & 10 & 11 & 12 \\
\hline Dates $\begin{array}{l}\text { Soir } \\
\text { Matin }\end{array}$ & $\begin{array}{l}3-9 \\
4-9\end{array}$ & $\begin{array}{l}4-9 \\
5-9\end{array}$ & $\begin{array}{l}5-9 \\
6-9\end{array}$ & $\begin{array}{l}6-9 \\
7-9\end{array}$ & $\begin{array}{l}7-9 \\
8-9\end{array}$ & $\begin{array}{l}8-9 \\
9-9\end{array}$ & $\begin{array}{r}9-9 \\
10-9\end{array}$ & $\begin{array}{l}10-9 \\
11-9\end{array}$ & $\begin{array}{l}11-9 \\
12-9\end{array}$ & $\begin{array}{l}12-9 \\
13-9\end{array}$ & $\begin{array}{l}13-9 \\
14-9\end{array}$ & $\begin{array}{l}14-9 \\
15-9\end{array}$ \\
\hline Heures Soir & $\begin{array}{r}21 \mathrm{H} \\
7 \mathrm{H}\end{array}$ & $\begin{array}{l}19 \text { н } \\
7 \text { н } \\
\end{array}$ & $\begin{array}{l}18 \mathrm{H} 50 \\
7 \mathrm{H}\end{array}$ & $\begin{array}{r}18 \times 55 \\
7 \text { H } 15\end{array}$ & $\begin{array}{l}19 \mathrm{H} \\
7 \mathrm{H} 05\end{array}$ & 18 H 55 & $\begin{aligned} & 19 \text { H } 15 \\
& 7 \text { H } 05\end{aligned}$ & $\begin{array}{r}18 \times 45 \\
7 \text { H } 15\end{array}$ & $\begin{array}{r}18 \text { H } 30 \\
7 \text { H } 15\end{array}$ & $\begin{array}{r}18 \text { н } 45 \\
7 \text { Н } 15\end{array}$ & $\begin{array}{r}18 \text { H } 50 \\
7 \text { H } 20\end{array}$ & $\begin{array}{r}18 \text { H } 50 \\
7 \text { H } 05\end{array}$ \\
\hline $\begin{array}{r}\text { Soir } \\
\text { rempératures } \\
\text { de 1'air } \\
\text { Matin } \\
\end{array}$ & & $\begin{array}{l}15,1^{\circ} \mathrm{C} \\
14,8^{\circ} \mathrm{C} \\
\end{array}$ & $\begin{array}{l}14,6^{\circ} \mathrm{C} \\
15,4^{\circ} \mathrm{C} \\
\end{array}$ & $\begin{array}{l}18,5^{\circ} \mathrm{C} \\
12,2^{\circ} \mathrm{C}\end{array}$ & $\begin{array}{l}20,5^{\circ} \mathrm{C} \\
17^{\circ} \mathrm{C}\end{array}$ & $\begin{array}{l}17,2^{\circ} \mathrm{C} \\
16,4^{\circ} \mathrm{C} \\
\end{array}$ & $18,8^{\circ} \mathrm{C}$ & $\begin{array}{l}15^{\circ} \mathrm{C} \\
10,5^{\circ} \mathrm{C}\end{array}$ & $\begin{array}{l}12,5^{\circ} \mathrm{C} \\
9,6^{\circ} \mathrm{C} \\
\end{array}$ & $14^{\circ} \mathrm{C}$ & $\begin{array}{l}11,9^{\circ} \mathrm{C} \\
10,6^{\circ} \mathrm{C} \\
\end{array}$ & $\begin{array}{l}16^{\circ} \mathrm{C} \\
14^{\circ} \mathrm{C} \\
\end{array}$ \\
\hline Hét éorologie & & $R \cdot X, O$ & ㅁ. *. 0 & $\square . \vee .=$ & $\square . v$. & $\square . \vee$, r & $\square . v$ & $\bullet ., x . \equiv$ & B.R. & $x-\cdot$ & •.*.*. & $\square . V . o$ \\
\hline $\begin{array}{l}\text { Espèces } \\
\text { capturées }\end{array}$ & & 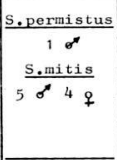 & 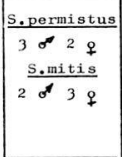 & $\begin{array}{c}\text { S.permistus } \\
\begin{array}{c}4 \delta q \\
\text { S.mitis } \\
1 \sigma^{d}\end{array}\end{array}$ & $\frac{\text { S.permistus }}{1 d^{2} q}$ & $\begin{array}{c}\frac{\text { S.permistus }}{3 d^{1} q} \\
\frac{\text { S.mitis }}{2 \sigma^{4} q} \\
\frac{\text { S.vibex }}{1 q}\end{array}$ & $\begin{array}{c}\frac{\text { S.permistus }}{2 \sigma^{\prime}} \\
\frac{\text { s.mitis }}{2 q}\end{array}$ & $\frac{\text { S.permistus }}{3 \sigma^{2} q}$ & $\begin{array}{c}\frac{\text { S.permistus }}{26 \sigma^{\prime} 33 q} \\
\frac{\text { S.mitis }}{18 \sigma^{7} q} \\
\frac{\text { M.fissa }}{1 \sigma^{4}}\end{array}$ & $\begin{array}{l}\text { S.permistus } \\
7 \begin{array}{l}16 q \\
\frac{\text { s.mitis }}{6} \\
8 \frac{\sigma^{\prime} 16 q}{q} \\
\text { M.testacea }\end{array} \\
1 \text { of }\end{array}$ & 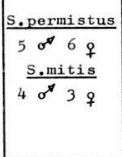 & 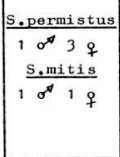 \\
\hline $\begin{array}{c}\text { Nombre } \\
\text { d'individus } \\
\text { marnués } \\
\text { recapturés }\end{array}$ & & 1 & & & 2 & & & & 3 & 2 & & \\
\hline
\end{tabular}

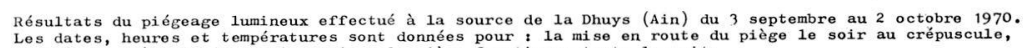

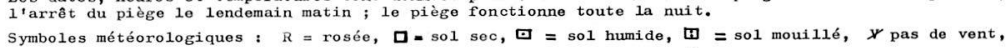
$v$ vent, v petite brise, - pluie, $\dot{\nabla}$ averse de pluie,

$$
\boldsymbol{\kappa} \text { orage, }=\text { brume, }=\text { brouillard, } 0 \text { beau temps. }
$$

(Remarque-) - Le piège lumineux a permis la capture d'autres espèces de Trichoptères que celles du "groupe 


\begin{tabular}{|c|c|c|c|c|c|c|c|c|c|c|c|c|c|}
\hline $\mathrm{N}^{0}$ piège $^{-1}$ & 13 & 14 & 15 & 16 & 17 & 18 & 19 & 20 & 21 & 22 & 23 & 24 & 25 \\
\hline \begin{tabular}{|c|} 
Soir \\
Dates \\
Natin \\
\end{tabular} & $\begin{array}{l}15-9 \\
16-9\end{array}$ & $\begin{array}{l}16-9 \\
17-9\end{array}$ & $\begin{array}{l}17-9 \\
18-9\end{array}$ & $18-9$ & $19-9$ & $20-9$ & $\begin{array}{l}21-9 \\
22-9\end{array}$ & $22-9$ & $23-9$ & $24-9$ & $29-9$ & 9-90 & $1-10$ \\
\hline \begin{tabular}{|c|}
$\begin{array}{c}\text { Soir } \\
\text { Heures } \\
\text { Matin }\end{array}$ \\
\end{tabular} & $\begin{array}{r}18 \times 30 \\
7 \times 15 \\
\end{array}$ & $\begin{array}{l}18 \times 40 \\
7 \mathrm{H} 15 \\
\end{array}$ & $\begin{array}{r}18 \text { \% } 30 \\
7 \mathrm{H} 05 \\
\end{array}$ & $\begin{array}{l}18 \mathrm{H} 30 \\
17 \mathrm{H} \\
\end{array}$ & $\begin{array}{l}18 \text { H } 15 \\
7 \text { H } 15 \\
\end{array}$ & $\begin{array}{l}19 \text { Н } 15 \\
7 \text { H }\end{array}$ & $\begin{array}{r}18 \text { H } 30 \\
7 \text { H } 15 \\
\end{array}$ & $\begin{array}{l}18 \text { H } 30 \\
7+15 \\
\end{array}$ & $\begin{array}{r}18 \text { \% } 30 \\
7+30\end{array}$ & $\begin{array}{r}18 \times 30 \\
7+30 \\
\end{array}$ & $\begin{array}{r}18 \mathrm{H} 30 \\
7+30 \\
\end{array}$ & $\begin{array}{r}18 \text { H } 15 \\
7+30 \\
\end{array}$ & $\begin{array}{l}18 \mathrm{H} \\
7 \mathrm{H}-15\end{array}$ \\
\hline \begin{tabular}{|c|}
\multicolumn{1}{|c|}{ So1r } \\
Tomp. \\
Mat $1 \mathrm{nn}$ \\
\end{tabular} & $\begin{array}{r}17,2^{\circ} \mathrm{C} \\
\quad 10,8^{\circ} \mathrm{C} \\
\end{array}$ & $\begin{array}{l}18,6^{\circ} \mathrm{C} \\
16,9^{\circ} \mathrm{C} \\
\end{array}$ & $\begin{array}{r}13,3^{\circ} \mathrm{C} \\
2,4^{\circ} \mathrm{C} \\
\end{array}$ & $\begin{array}{l}14^{\circ} \mathrm{C} \\
16,1^{\circ} \mathrm{C} \\
\end{array}$ & $\begin{array}{r}14,5^{\circ} \mathrm{C} \\
12,3^{\circ} \mathrm{C} \\
\end{array}$ & $\begin{array}{l}15,6^{\circ} \mathrm{C} \\
13,1^{\circ} \mathrm{C} \\
\end{array}$ & $\begin{array}{l}17.3^{\circ} \mathrm{C} \\
14{ }^{\circ} \mathrm{C}\end{array}$ & $\begin{array}{l}17,8^{\circ} \mathrm{C} \\
13,2^{\circ} \mathrm{C} \\
\end{array}$ & $\begin{array}{l}15,2^{\circ} \mathrm{C} \\
10,1^{\circ} \mathrm{C}\end{array}$ & $\begin{array}{l}14^{\circ} \mathrm{C} \\
10^{\circ} \mathrm{C} \\
\end{array}$ & $\begin{array}{l}15,7^{\circ} \mathrm{C} \\
13^{\circ} \mathrm{C}\end{array}$ & $\begin{array}{r}13,2^{\circ} \mathrm{C} \\
11,2^{\circ} \mathrm{C} \\
\end{array}$ & $\begin{array}{l}10,2^{\circ} \mathrm{C} \\
8,5^{\circ} \mathrm{C} \\
\end{array}$ \\
\hline \begin{tabular}{|r|} 
Mét tóoro- \\
10gio \\
\end{tabular} & 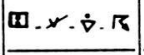 & ㅁ.. 0 & ロ.*. & 口.X. 0 & ๑.*. 0 & $R \cdot \forall .=$ & 口. & $\square * x=$ & ๑. ‥ $=$ & G.R.X. O & ㅁ. $* 0$ & 四. & 四. $\forall$. \\
\hline $\begin{array}{l}\text { Espèces } \\
\text { capturéos }\end{array}$ & $\begin{array}{c}\frac{\text { S.pormistus }}{209 q} \\
\frac{\text { S,mitis }}{27 \sigma^{\prime 25} q} \\
\frac{\text { M, impunctatus }}{10}\end{array}$ & $\begin{array}{c}\frac{\text { S.permistus }}{12 \sigma^{2} 8} \\
\frac{\text { s,mitis }}{4 \sigma^{2} q} \\
\frac{M, \text { estacea }}{1 q}\end{array}$ & 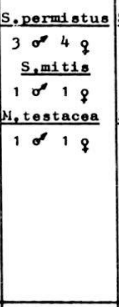 & 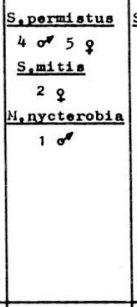 & $\frac{\text { S.permistus }}{2 \text { on } 1 q}$ & $\frac{\text { s,permistus }}{1 q}$ & $\left|\begin{array}{c}\text { S.permistus } \\
1012 q \\
\frac{\text { S,mitis }}{1012 q} \\
\frac{\text { Meimpunctatus }}{1 q}\end{array}\right|$ & $\begin{array}{c}\frac{\text { S.permistus }}{1 \sigma^{2} q} \\
\frac{\text { s,mitis }}{9 q} \\
\frac{s, \text { vibex }}{1 \sigma}\end{array}$ & 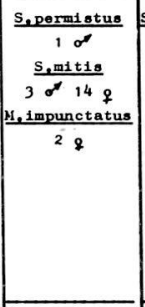 & \begin{tabular}{|} 
S,permistus \\
$2 \sigma^{4} q$ \\
$\frac{\text { s,mitis }}{7 q}$ \\
$\frac{\text { s,ribox }}{2 \sigma^{\prime}}$
\end{tabular} & \begin{tabular}{|c|}
$\frac{\text { s.permistus }}{2 q}$ \\
$\frac{\text { S,mitis }}{12047 q}$ \\
$\frac{\text { S.vibox }}{100}$ \\
$\frac{M, \text { fissa }}{1 q}$ \\
$\frac{\text { M, sequax }}{10}$ \\
10 impunctatus \\
10 \\
\end{tabular} & 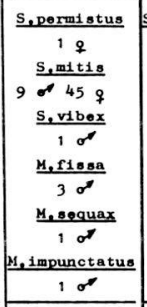 & $\begin{array}{l}\text { S.permistus } \\
\frac{2 q}{20 \%} \\
\frac{\text { s,mitis }}{20}\end{array}$ \\
\hline \begin{tabular}{|c|} 
Nombro \\
d'lnd. \\
marqués \\
cecapturés
\end{tabular} & 1 & & & 1 & & & & & & & & & \\
\hline
\end{tabular}

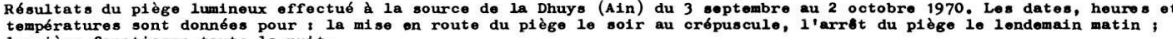

10 proge ronctionne toute la nalt.

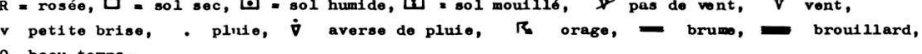

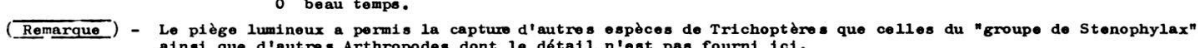

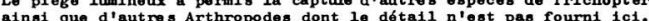

Fig. 5. Tableau récapitulatif des résultats du piégeage lumineux. 
Les femelles recapturées présentent toutes des ovaires mûrs et sont ainsi prêtes. pour la ponte. Il est donc logique de conclure à la sortie des Trichoptères qui, de la grotte où ils séjournent durant l'été, prennent leur vol pour aller pondre dans les ruisseaux épigés.

$\mathrm{Au}$ cours de cette expérience, nous notons tout d'abord la répartition des sexes dans les captures de Trichoptères (fig. 3); pendant la première quinzaine du mois de septembre, les mâles et les femelles sont capturés en nombre identique; à partir du 19 septembre, les mâles deviennent moins nombreux, alors que le nombre des femelles augmente pour devenir plus de trois fois supérieur à celui des mâles les 29-30 septembre. Ceci est remarquable si l'on se souvient que les mêmes espèces de Trichoptères, sous terre, présentent un sex-ratio inverse, avec une nette dominance des mâles par rapport aux femelles (Bouvet et Ginet, 1969).

D'autre part, les conditions météorologiques enregistrées simultanément ne semblent pas révéler une influence particulière de la température sur le nombre de captures de Trichoptères (Fig. 5). Le maximum des captures correspond aux nuits à vent faible ou nul, lorsque l'humidite du sol et de l'air (rosée ou pluie) est importante (fig. 4).

En conclusion, la capture des Trichoptères du "groupe de Stenophylax" à l'aide d'un piège lumineux permet de mettre en évidence:

- leur sortie des grottes, pour les mâles comme pour les femelles;

- la capacité de ponte des femelles au moment de leur migration.

\section{RÉ S U M É}

Les Trichoptères du "groupe de Stenophylax" se rencontrent très fréquemment dans les grottes. Jusqu'à présent, à la suite de Jeannel (1926), les auteurs semblaient n'accorder à ce séjour que la valeur d'un hasard, ces Insectes, entraînés sous terre, devant y périr.

A l'aide de la méthode du piège lumineux, il est maintenant démontré que les Trichoptères cavernicoles, non seulement séjournent sous terre tout l'été, mais qu'à l'automne (septembre-octobre) ils en ressortent activement pour aller pondre dans les ruisseaux épigés.

\section{Z U S A M M E N F A S S U N G}

Die "Trichopteren" der "Stenophylax-Gruppe" sind sehr oft in Höhlen zu finden. Bis heute schienen die Autoren nach Jeannel (1926) diesen Aufenthalt nur dem Zufall zuzuschreiben, denn diese Insekten mussten, nachdem sie unter die Erde gelenkt wurden, dort sterben.

Mit Hilfe der "Lichtfallen-Methode" wird demonstriert, dass die HöhlenTrichopteren sich nicht nur im Sommer unter der Erde aufhalten, sondern dass sie im Herbst (September, Oktober) sehr aktiv daraus hervorkommen, um in den Bächen draussen Eier zu legen. 


\section{B I B L I O G R A P H I E}

BITSCH, J. et FROCHOT, B. 1962. Données récentes sur les Trichoptères cavernicoles. Spelunca, Mémoires, 4e S., no. 2, 64-70.

BOURNAUD, M., GAUTHERON-DURANTHON, F. 1969. Essai d'étude d'une population de Trichoptères cavernicoles dans la grotte du Crochet (Ain) par la méthode des captures-recaptures. Bull. Soc. Zool. Fr., 94, no. 3, 471-483.

BOUVET, Y. 1970. Ecologie et Biologie des Trichoptères cavernicoles. Thèse 3ème cycle, Lyon, $57 \mathrm{p}$.

BOUVET, Y., GINET, R. 1969. Données biologiques et biogéographiques sur le "groupe de Stenophylax" cavernicoles en France (Insectes, Trichoptères). Bull. Soc. Linn. Lyon., no. $10,334-349$.

FONTAINE, J. 1971. Un piege lumineux de terrain utilisable pour la capture d'insectes à larves aquatiques. Bull. Soc. Linn. Lyon, sous presse.

JEANNEL, R. 1926. Faune cavernicole de la France. Lechevalier ed. Paris, 334 p.

JEANNEL, R. 1943. Les fossiles vivants des cavernes. Gallimard, Paris, $321 \mathrm{p}$.

MOTAS, C., DECOU, V., BURGHELE, A. 1967. Sur 1'association pariétale des grottes d’Olténie (Roumanie). Ann. Spéléo., XXII, 3, 475-522. 